\title{
Optimal Imaging Positions for 3-Phase Bone Scanning of Patients with Bony Pathology of the Feet
}

\author{
Mona M. Baqer and Issa Loutfi \\ Department of Nuclear Medicine, Faculty of Medicine, Kuwait University, Safat, Kuwait
}

\begin{abstract}
The aim of the study was to optimize imaging positions of ${ }^{99 m T c-}$ methylene diphosphonate ( ${ }^{99 m}$ Tc-MDP) 3-phase bone scanning for the accurate localization of foot pathology in patients with trauma and diabetes-related complications. Methods: ${ }^{99 m T c-}$ MDP 3-phase bone scanning was performed for 26 controls and 27 patients with foot pathology. Flow was acquired in 1 of the following projections: anterior-posterior, medial-lateral, or plantar. Blood-pool and delayed images were acquired in a set of 5 projections (anterior, posterior, medial, lateral, and plantar). Images from the control group were checked for the views that best visualized individual bones or regions of the foot. These views were cross-correlated with images from the patient group to see whether they localized the exact site of the foot lesion. Results: In the controls, the plantar view was the best view for visualization of the forefoot region. The mid foot was best assessed on the anterior view. Medial-lateral views were best suited for imaging the hind foot, and the posterior view was the best for the ankle joint. In the subjects with foot pathology, lesions were accurately assigned to the affected bone using the imaging criteria derived from the controls. In a few cases, however, additional views were needed because of overlap or shine-through of activity, particularly in mid-foot lesions. Conclusion: Optimal imaging positioning of the foot by bone scanning can be achieved using 5 views, which can yield accurate localization of a particular structure or bone, thereby improving the diagnostic accuracy of the procedure.
\end{abstract}

Key Words: foot; bone scan; diabetes; osteomyelitis; fracture

J Nucl Med Technol 2010; 38:69-75

DOI: 10.2967/jnmt.109.070771

$\mathbf{T}$ he evaluation of foot pathology using imaging techniques can be challenging because of the presence of various structures near each other, including many bones with irregular interdigitations and multiple small joints. In addition, different parts of the foot can be affected, depending on the disease process involved, requiring image acquisition to

Received Sep. 30, 2009; revision accepted Mar. 15, 2010.

For correspondence or reprints contact: Issa Loutfi, Department of Nuclear Medicine, Faculty of Medicine, Kuwait University, P.O. Box 24923, 13110 Safat, Kuwait.

E-mail: loutfi@hsc.edu.kw

COPYRIGHT @ 2010 by the Society of Nuclear Medicine, Inc. be directed more specifically to that area and the imaging process to be optimized for the problem at hand. For example, the metatarsal bones and proximal phalanges are the bones mostly affected by osteomyelitis in diabetic patients (1), and stress fractures occur more commonly in the talus and calcaneus (2). Currently, imaging strategies of foot conditions involve the use of multiple imaging modalities, usually starting with conventional radiography and followed by radionuclide scanning, CT, or MRI. Each modality has its own merits, and its use depends on many factors, including the condition under study, patient and referring physician's preference, and availability.

Bone scintigraphy using ${ }^{99 \mathrm{~m}} \mathrm{Tc}$-labeled diphosphonate compounds is a common procedure for the diagnosis of abnormalities involving the foot. Thanks to its high sensitivity for metabolic changes, which usually precede anatomic changes, the bone scan often detects lesions early in their development. In addition, bone scintigraphy provides physiologic information about certain structural abnormalities to determine their clinical significance (3). Currently, the bone scan plays a key role in the detection of acute and chronic injuries seen in athletes, such as stress fractures, enthesopathies, and avulsion lesions (4), and the evaluation of inflammatory conditions, such as osteomyelitis and diabetic foot complications.

Technically, bone scanning is a well-established procedure; however, positions for imaging the feet are not fully standardized yet. The use of appropriate foot positioning is crucial to bone scintigraphy to identify the site of bony involvement and discriminate between the affected structures. Highresolution images acquired according to the anatomic layout of the bones in the region studied would minimize overlap of these bones, thus providing a better evaluation of the lesions present $(5,6)$. The objective of this study was to optimize foot positioning in ${ }^{99 \mathrm{~m}} \mathrm{Tc}$-methylene diphosphonate ( ${ }^{99 \mathrm{~m}} \mathrm{Tc}-\mathrm{MDP}$ ) bone scanning for identifying pathology of various etiologies in different bones and areas of the foot.

\section{MATERIALS AND METHODS}

We prospectively studied 53 subjects selected from a group of patients referred for bone scintigraphy at the Nuclear Medicine Department between February and October 2006. The subjects 
TABLE 1

Demographics of Subjects Studied

\begin{tabular}{lcc}
\hline Demographic & Controls & Patients \\
\hline Average age \pm SD $(y)$ & $49 \pm 20$ & $45 \pm 17$ \\
Sex $(n)$ & & \\
Male & 10 & 16 \\
$\quad$ Female & 16 & 11 \\
Clinical condition $(n)$ & - & \\
Diabetes & - & 14 \\
Trauma & - & 5 \\
Arthritis & - & 4 \\
Foot pain & 5 & - \\
Back pain & 21 & - \\
\hline Cancer (breast, prostate, or other) & & \\
\hline
\end{tabular}

were divided into the following 2 groups: controls-comprising 26 patients, referred for complaints not related to the feet-and patients-comprising 27 patients, referred from the diabetic or orthopedic clinics for evaluation of foot pathology including diabetic foot, trauma, arthritis, and pain.

The demographic details of the groups studied are shown in Table 1. All subjects gave their informed consent for participating in the study, which did not alter their subsequent management. The study was approved by the institutional ethics committee.

On arrival, a history and physical examination were acquired for each patient. Lists of relevant laboratory and imaging studies were recorded on a data sheet. A 3-phase bone scan was obtained as follows (7).
For the controls, the flow phase was acquired after positioning the feet in anterior-posterior (10 patients), lateral-medial (10 patients), or plantar ( 6 patients) projections. For the patients, the flow position of the feet was selected on the basis of the findings in the control group as to which position would optimize the assessment of flow in the lesion under evaluation.

For the anterior-posterior views, feet were positioned with the patients lying supine on the imaging table. The patients were asked to have their feet apart in a neutral (dropping) anterior position, with a slight internal rotation of the ankle joints. Positioning aids such as small cushions and adhesive tape were used to keep the feet still and prevent an unintentional change of position during acquisition while keeping the patients comfortable at the same time. The first head of the scintillation camera was then placed over the feet and ankle region parallel to the imaging table and as close as possible to the feet for the anterior view. The second head was placed under and parallel to the imaging table for the posterior view.

The medial and lateral views of the feet were obtained by placing the patients in the frog's-leg position, with their heels held close together. The first head of the scintillation camera was then placed over the feet and ankle region parallel to the imaging table and as close as possible to the feet for the medial view, and the second head was placed under and parallel to the imaging table for the lateral view.

The plantar view was obtained by placing the feet directly on 1 camera head, with the patients supine or sitting.

After patient positioning, ${ }^{99 \mathrm{~m}} \mathrm{Tc}-\mathrm{MDP}$ (740-1,110 MBq [20-30 $\mathrm{mCi}]$ ) was injected intravenously as a bolus through an indwelling

TABLE 2

Best Views for Specific Foot Regions and Bones from Control Group

\begin{tabular}{|c|c|c|c|c|c|c|}
\hline \multirow[b]{3}{*}{ Region of foot } & \multicolumn{6}{|c|}{ Phase of bone scan } \\
\hline & \multicolumn{2}{|c|}{ Flow } & \multicolumn{2}{|c|}{ Blood pool } & \multicolumn{2}{|c|}{ Delayed } \\
\hline & Projection & Score & Best view & Score & Best view & Score \\
\hline Forefoot & Plantar & 6 & & & & \\
\hline \multirow[t]{2}{*}{ Phalanges } & & & Plantar & 18 & Plantar & 15 \\
\hline & & & Anterior & 3 & Anterior & 4 \\
\hline \multirow[t]{2}{*}{ Metatarsals } & & & Plantar & 15 & Plantar & 14 \\
\hline & & & Anterior & 5 & Anterior & 5 \\
\hline \multirow[t]{2}{*}{ MPJs } & & & Plantar & 17 & Plantar & 15 \\
\hline & & & Anterior & 3 & Anterior & 3 \\
\hline Mid foot & Anterior & 10 & & & & \\
\hline \multirow[t]{3}{*}{ Cuneiform bones } & & & Anterior & 19 & Anterior & 18 \\
\hline & & & Plantar & 5 & Plantar & 4 \\
\hline & & & Medial & 1 & Medial & 1 \\
\hline \multirow[t]{3}{*}{ Cuboid bone } & & & Anterior & 22 & Anterior & 23 \\
\hline & & & Plantar & 1 & Plantar & 2 \\
\hline & & & Medial & 1 & Medial & 1 \\
\hline \multirow[t]{3}{*}{ Navicular bone } & & & Anterior & 22 & Anterior & 23 \\
\hline & & & Plantar & 2 & Plantar & 1 \\
\hline & & & Medial & 2 & Medial & 1 \\
\hline Hind foot & Medial-lateral & 10 & & & & \\
\hline \multirow[t]{3}{*}{ Calcaneus } & & & Medial-lateral & 17 & Medial and lateral & 20 \\
\hline & & & Lateral & 4 & Lateral & 4 \\
\hline & & & Posterior & 1 & Posterior & 2 \\
\hline \multirow[t]{2}{*}{ Talus } & & & Medial-lateral & 19 & Medial and lateral & 23 \\
\hline & & & Lateral & 1 & Lateral & 1 \\
\hline Ankle & Posterior & 10 & & & & \\
\hline Ankle joint & & & Posterior & 26 & Posterior & 26 \\
\hline
\end{tabular}


TABLE 3

Scan Findings in Patient Group

\begin{tabular}{|c|c|c|c|c|c|c|}
\hline \multirow[b]{3}{*}{ Region of foot } & \multicolumn{6}{|c|}{ Phase of bone scan } \\
\hline & \multicolumn{2}{|c|}{ Flow } & \multicolumn{2}{|c|}{ Blood pool } & \multicolumn{2}{|c|}{ Delayed images } \\
\hline & Projection & $\bar{n}$ & Best view & $\bar{n}$ & Best view & $n$ \\
\hline \multicolumn{7}{|l|}{ Forefoot } \\
\hline \multirow[t]{3}{*}{ Phalanges } & Anterior & 3 & Anterior & 6 & Anterior & 5 \\
\hline & & & Posterior & 1 & Posterior & 1 \\
\hline & & & Plantar & 3 & Plantar & 4 \\
\hline \multirow[t]{2}{*}{ Metatarsals } & Anterior & 1 & Anterior & 3 & Anterior & 5 \\
\hline & & & Plantar & 2 & Plantar & 8 \\
\hline \multirow[t]{3}{*}{ MPJs } & Anterior & 2 & Anterior & 8 & Anterior & 9 \\
\hline & & & Posterior & 2 & Posterior & 1 \\
\hline & & & Plantar & 5 & Plantar & 10 \\
\hline \multicolumn{7}{|l|}{ Mid foot } \\
\hline \multirow[t]{2}{*}{ Cuneiform bones } & Anterior & 3 & Anterior & 2 & Anterior & 3 \\
\hline & & & Plantar & 2 & Medial & 1 \\
\hline \multirow[t]{2}{*}{ Cuboid bone } & Anterior & 3 & Anterior & 1 & Anterior & 3 \\
\hline & & & Plantar & 1 & Plantar & 3 \\
\hline \multirow[t]{2}{*}{ Navicular bone } & Anterior & 2 & Anterior & 1 & Anterior & 2 \\
\hline & & & Posterior & 1 & Posterior & 1 \\
\hline \multicolumn{7}{|l|}{ Hind foot } \\
\hline \multirow[t]{2}{*}{ Calcaneus } & Medial-lateral & 2 & Medial & 3 & Medial & 4 \\
\hline & & & Lateral & 3 & Lateral & 3 \\
\hline \multirow[t]{3}{*}{ Talus } & & & Medial & 1 & Medial & 2 \\
\hline & & & & & Lateral & 3 \\
\hline & & & & & Posterior & 1 \\
\hline \multicolumn{7}{|l|}{ Ankle } \\
\hline & Medial-lateral & 1 & Medial & 1 & Medial & 1 \\
\hline \multirow[t]{2}{*}{ Ankle joint } & & & Lateral & 2 & Lateral & 2 \\
\hline & & & Posterior & 2 & Posterior & 2 \\
\hline
\end{tabular}

line. Dynamic images $(20$ frames at $3 \mathrm{~s}$ per frame using a $64 \times 64$ pixel matrix) were acquired using a dual-head scintillation camera (Millenium; GE Healthcare) equipped with a low-energy highresolution parallel-hole collimator. A $20 \%$ energy window centered on $140 \mathrm{keV}$ was used. Spot blood-pool views of the feet were obtained in a $256 \times 256$ matrix and were acquired for all patients in 3 positions (anterior-posterior, medial-lateral, and plantar) for $2 \mathrm{~min}$ each. Spot delayed images of the feet were obtained at $3 \mathrm{~h}$ after injection in the same positions as the blood-pool images for 5 min each. In addition, whole-body blood-pool and delayed images were acquired. Additional views and a SPECT scan were obtained as needed to address the clinical question at hand.

In the controls, the acquired sets of images of the feet for each subject were reviewed simultaneously by 2 senior nuclear medicine physicians with the objective of recording the observed structures and of deciding on the best view identifying individual bones. This was done using a worksheet on which the foot was divided into 4 anatomic regions: forefoot, mid foot, hind foot, and ankle joint. Under each region, the individual bones, or groups of similar bones present anatomically, were listed in a table format (Table 2). Once a set of foot images had been inspected, the reviewers recorded the view or views that best showed each individual bone for the bloodpool and delayed images-for example, anterior, plantar, and medial for the navicular bone. The best flow views that clearly identified a particular foot region (forefoot, mid foot, etc.) were recorded, because the flow images lacked the details to identify individual bones. The process of assigning the best views for each individual bone was repeated for all 26 controls. Each time a view was identified in the way described, a score of 1 was given to it and added to existing scores from the previous set of images.

In the patients, the images were inspected by the same physicians for abnormalities of flow, blood pool, and delayed uptake in the feet and the structures involved. In particular, the anatomic location and pattern of the abnormalities present were documented. In a way similar to that for the control-group images, each abnormality present was tabulated, along with the view identifying it best every time it was seen (Table 3). Cross-reference was made with the findings in the control group to check whether the best views for a particular foot pathology corresponded to the predicted ones from the controls.

\section{RESULTS}

An example of the image set is displayed in Figure 1. The results of scan review in the control group are shown in Table 2. The plantar view was the best view for forefoot evaluation in a significant number of subjects (18/26 [69\%]). The plantar view showed best the phalanges in 15 subjects, metatarsals in 14 subjects, and metatarsophalangeal joints (MPJs) in 15 subjects. For the mid foot, the anterior view was the best for showing the cuneiform bones in 18 of $26(69 \%)$ subjects and the cuboid and navicular in 23 of $26(88 \%)$ subjects. The hind-foot bones were best seen on medial-lateral views for 17 of $26(65 \%)$ subjects. The posterior view was the best for the ankle joint in 20 of $26(77 \%)$ subjects. 


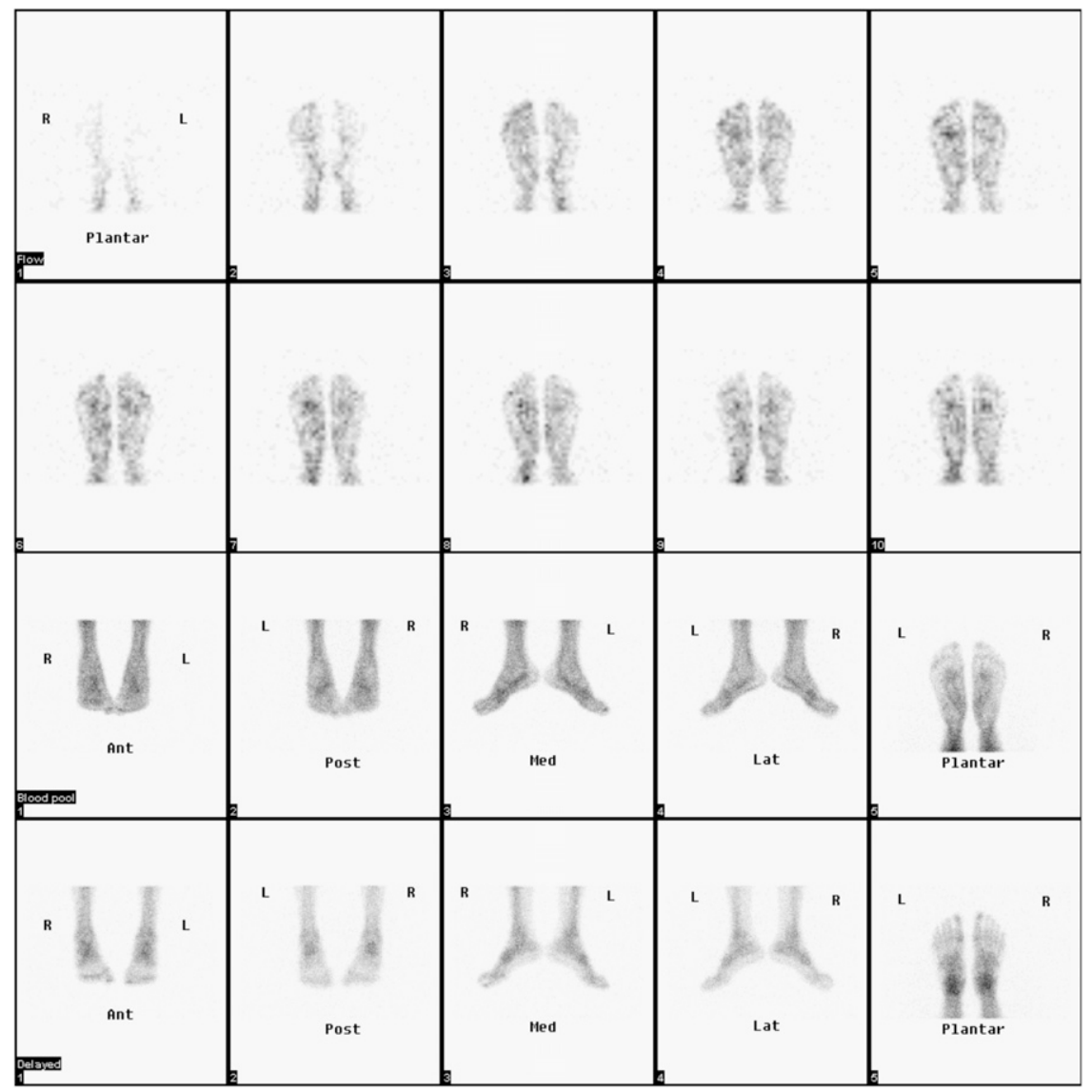

FIGURE 1. Three-phase bone study in control. Upper 2 rows display sequential 3-s/frame flow images acquired in plantar position. Third row shows blood-pool images, and in fourth row, corresponding delayed images in 5 positions are shown. Ant $=$ anterior; Post $=$ posterior; Med $=$ medial; Lat $=$ lateral.

The bone scan results in the patient group are shown in Table 3. Among the 27 patients, 17 (63\%) had forefoot pathology, 7 (26\%) mid-foot pathology, 11 (41\%) hind-foot pathology, and $4(15 \%)$ ankle joint pathology. The flow view was helpful in indicating the site of lesions in 17 patients. For planar imaging, the anterior view was the best for phalanx pathology in 5 of 7 patients, metatarsal lesions in 5 of 9 patients, and MPJ lesions in 9 of 10 patients (Fig. 2). The plantar view was the best for metatarsal and MPJ lesions in 8 of 9 and 10 of 10 patients, respectively. The anterior view was the best for all 3 patients with cuneiform and cuboid lesions and 2 of 4 patients with navicular bone pathology (Fig. 2). The medial-lateral view was the best for 4 of 7 and 2 of 4 patients with calcaneus and talus pathology, respectively (Fig. 3). The posterior view was the best for 2 of 4 patients with ankle joint pathology.

The clinical and radiographic evaluation of the patients showed that on the plain radiograph, 14 of 27 patients had foot pathology related to diabetes, and of these 9 had forefoot pathology (6 metatarsal, 4 phalanges, and 3 MPJ lesions). Five diabetic subjects had mid-foot pathology (2 cuboid, 2 navicular, and 2 cuneiform lesions). Three patients in this group had Charcot joint in mid foot, talus, and ankle joint. Five of 27 had a traumatic foot lesion. A stress fracture in the navicular bone was found in 1 patient. A calcaneus spur was seen in 1 patient, and 3 patients presented with a fracture in the metatarsal and MPJ. Four patients had minor arthritic changes shown on radiography, and in the remaining 4 patients complaining of foot pain, no obvious radiographic abnormalities in the feet were observed.

In addition to pathologic causes, areas of increased uptake could also have been due to position artifacts. For example, in medial-lateral views focal areas of increased uptake in the forefoot bilaterally at the MPJ region were observed. In the posterior and medial views, areas of diffuse increased uptake in the mid foot due to overlap of bones were found. Also, in the anterior-posterior views increased metatarsal uptake was noted bilaterally, which was position-related. These findings are summarized in Table 4 and are displayed in Figure 1. Artifacts were seen also in the patient group, mainly because of superimposition of the bones and shine-through of activity from affected bones. Hind-foot bones were superimposed on the posterior and plantar views. In the medial-lateral views, the forefoot and mid-foot bones were superimposed. Lesions in the talus overlapped the navicular bone and made it difficult to identify in the lateral view, and lesions of the calcaneus overlapped the talus in the plantar view. These findings are summarized in Table 4.

A summary of the best views for foot scintigraphy based on the observations from this study is shown in Table 5 . 


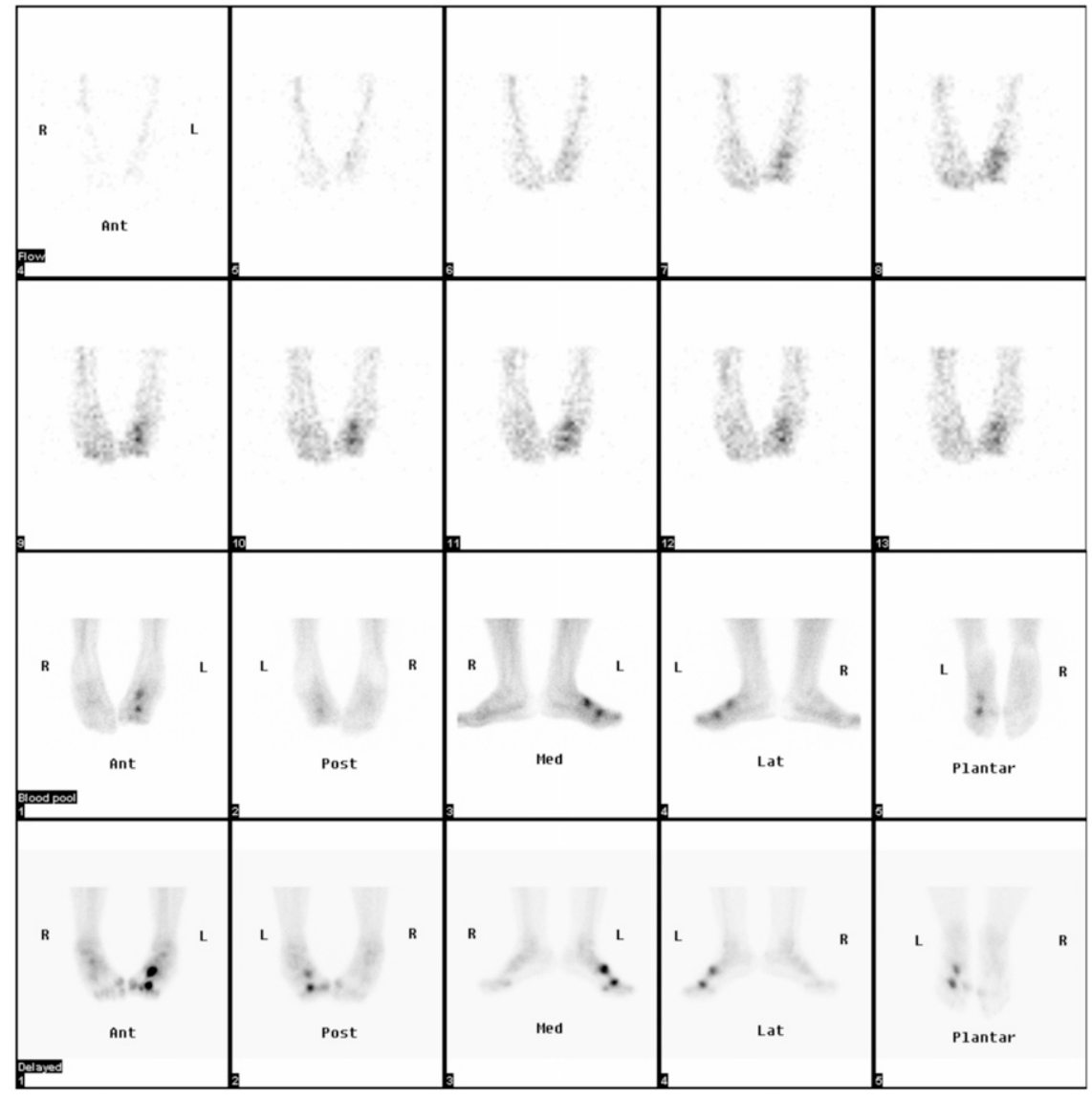

FIGURE 2. Three-phase bone scan in patient with trauma to left foot. Upper 2 rows display sequential 3-s/frame flow images acquired in anterior position, showing increased flow to mid left foot. Third row shows blood-pool images, and in fourth row, corresponding delayed images in 5 positions are shown. Anterior blood-pool and delayed views show clearly 2 abnormalities in third metatarsal bone, indicating fractures. Small tarsal bones are also better seen on these views. Ant $=$ anterior; Post $=$ posterior; Med $=$ medial; Lat $=$ lateral.

\section{DISCUSSION}

Foot pathology is frequently encountered in medical practice and can involve different parts of the foot $(8-10)$. The current diagnostic work-up of foot abnormalities frequently requires the use of imaging procedures including radiography, bone scanning, $\mathrm{CT}$, and others to detect various underlying changes, leading to appropriate management of the condition.

The real value of bone scintigraphy is that it can provide physiologic information about the structural abnormalities present. Furthermore, a 3-phase (or multiphase) bone scan can give information on various aspects of pathophysiology such as increased blood flow and blood pool, which reflect the degree of vascularity and activity of the lesion. The delayed spot views show uptake in the bone itself and identify the location of the lesion. However, because radionuclide imaging is not primarily an anatomic study because of the inherent problem of resolution, in addition to the large number and superimposition of the bones and structures of the foot, it is difficult to separate the bones and show the exact sites of lesions on a standard bone scan. Accurate localization of foot pathology on bone scans is challenging because of these reasons $(3,6)$; therefore, additional care in positioning the foot is needed for better identification of the lesions present. This situation is unlike musculoskeletal radiology, for which standard positioning and changing of the angles of each position show different structures and individual bones of the foot.

In this study, the aim was to optimize the imaging position of 3-phase bone scans for pathology of different bones and areas of the foot.

First, the normal anatomy of the pedal bones was studied in a 5-image set of the feet taken as part of the bone scan in controls. This set was to select the best view (or views) for the visualization of different areas and bones present. Next, these views were used as points of reference in the patient group to check whether they gave a better localization of the lesions present and thus improved the diagnostic accuracy of the test. One position (2 views for the anterior-posterior and mediallateral, 1 view for plantar) could be obtained for the flow study and was chosen according to the site of the lesion, as indicated by the clinical presentation and radiographic finding (11).

Optimal positions for the different parts of the foot were established. In the patients with foot pathology involving the forefoot, the plantar view showed pathology in most cases; however, the anterior view was considered equally good in a significant number. For hind-foot pathology, medial and lateral views were considered more appropriate (12). For mid-foot pathology, a range of views was considered optimal, including a combination of anterior and plantar views. 
FIGURE 3. Three-phase bone scan in patient with bilateral heel pain. Upper 2 rows display sequential 3-s/frame flow images acquired in medial position, showing increased flow to hind-foot areas. Third row shows blood-pool images, and in fourth row, corresponding delayed images in 5 positions are shown. Medial-lateral blood-pool and delayed views show clearly increased uptake in calcaneus bilaterally. Positive findings are also seen on posterior and plantar views. Calcaneal spurs were found on radiographs. Ant $=$ anterior; Post $=$ posterior; Med $=$ medial; Lat $=$ lateral.

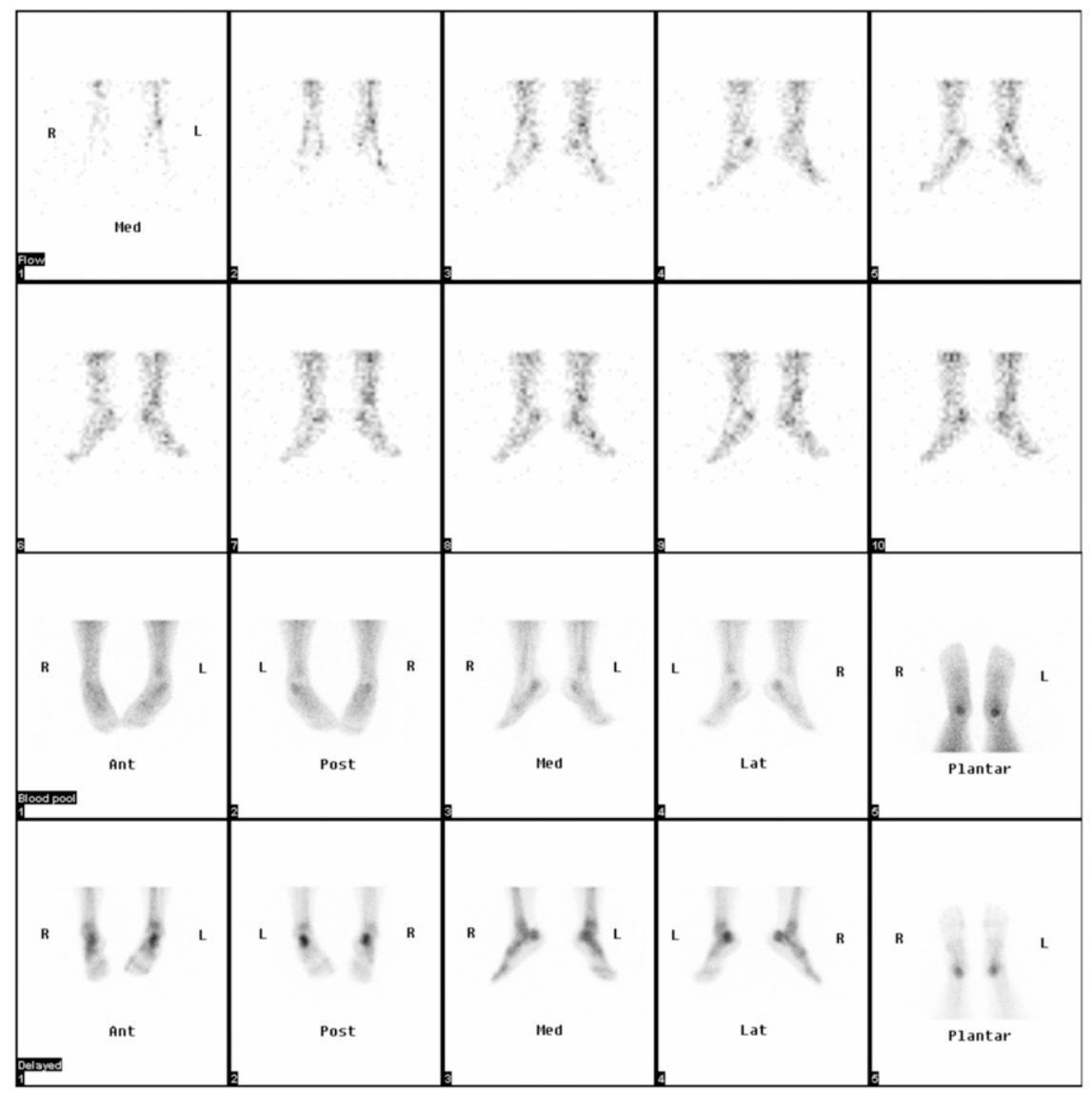

The other problem faced in bone scanning came from superimposition and shine-through of activity from various parts of the foot. This is particularly true for the mid foot. Again, acquisition of a set of views including anteriorposterior, plantar, and medial-lateral helped resolve the issue of superimposed activity and avoid misinterpretation of the images because of the positioning artifacts listed in Table 4.

Finally, optimal interpretation of the bone scan for foot pathology was also dependent on the bone involved and pattern of uptake. When uptake was symmetric and uniform, individual identification was not difficult. However, the presence of pathology commonly involved superimposition and shine-through of activity in affected bones. This was particularly true for the mid foot, for which additional views were necessary.

The summary of the best views, based on the observations from this study (Table 5), gives a rough guideline of foot imaging as part of a ${ }^{99} \mathrm{~m}$ Tc-MDP bone scan. A standard protocol involving acquisition of 5 views - anterior, posterior, medial, lateral, and plantar - would be adequate for optimal evaluation of foot pathology because of various causes.

Because we evaluated the 5-view approach, all patients with foot pathology who were referred for a bone scan have been routinely imaged using the protocol described in this study. This consistency has resulted in a more streamlined practice and a more efficient diagnostic service in our department.

TABLE 4

Position-Related Artifacts

\begin{tabular}{lll}
\hline \multicolumn{1}{c}{ View } & Scintigraphic appearance & Impact on interpretation \\
\hline Anterior-posterior (blood pool) & Focal increased metatarsal uptake & False-positive blood-pool image \\
Medial or lateral & Focal increased uptake in forefoot & Difficulty in separating bones of forefoot \\
Posterior & Diffuse increased uptake in mid and hind foot & Overlapping lesions in talus and navicular \\
& Diffuse increased uptake in mid foot & Difficulty in separating bones of mid foot \\
Plantar & Diffuse increased uptake in hind foot & Cannot differentiate talus and calcaneus \\
\hline
\end{tabular}


TABLE 5

Best View to Evaluate Specific Foot Region on 3-Phase Bone Scan

\begin{tabular}{lc}
\hline Foot region & \multicolumn{1}{c}{ Best view } \\
\hline Forefoot & $\begin{array}{c}\text { Plantar for flow, blood-pool, and } \\
\text { delayed images; anterior will be } \\
\text { useful to resolve overlap in } \\
\text { some cases } \\
\text { Anterior for all phases; lateral or } \\
\text { plantar will help in cases in } \\
\text { which cuneiform bones are } \\
\text { involved } \\
\text { Lid foot }\end{array}$ \\
Hind foot & foral and medial will be useful \\
Ankle joint & Posterior, medial, and lateral \\
\hline
\end{tabular}

\section{CONCLUSION}

Optimal views for different parts of the foot and ankle can be obtained by acquisition of 5 standardized planar views. Using this protocol, accurate localization of foot pathology on bone scanning can be achieved.

\section{REFERENCES}

1. Resnick D, Niwayama A. Diagnosis of Bone and Joint Disorders. Philadelphia, PA: WB Saunders; 1995.

2. Van der Wall H, Storey J, Frater C, Murray P. Importance of positioning and technical factors in anatomic localization of sporting injuries in scintigraphic imaging. Semin Nucl Med. 2001;31:17-27.

3. Ryan PJ, Fogelman I. The role of nuclear medicine in orthopaedics. Nucl Med Commun. 1994;15:341-360.

4. Minoves M. Bone and joints sports injuries: the role of bone scintigraphy. Nucl Med Commun. 2003;24:3-10.

5. Frater CJ. Importance of positioning and frequency of unexpected scintigraphic findings in a high impact sport. Nucl Med Commun. 2001;22:1231-1235.

6. Brown ML, O'Connor MK, Hung JC, Hayostek RJ. Technical aspects of bone scintigraphy. Radiol Clin North Am. 1993;31:721-730.

7. Donohoe KJ, Henkin RE, Royal HD, et al. Procedure guideline for bone scintigraphy: 1.0. Society of Nuclear Medicine. J Nucl Med. 1996;37:19031906.

8. Elgazzar AH, Abdel-Dayem HM. Imaging skeletal infections: evolving considerations. In: Freeman LM, ed. Nuclear Medicine Annual. Philadelphia, PA: Lippincott Williams \& Wilkins; 1999.

9. Gold RH, Tong DJ, Crim JR, Seeger LL. Imaging the diabetic foot. Skeletal Radiol. 1995;24:563-571.

10. Monteleone GP Jr. Stress fractures in the athlete. Orthop Clin North Am. 1995;26:423-432.

11. Lewis P. Scintigraphy in the foot and ankle. Foot Ankle Clin. 2000;5:1-27.

12. Groshar D, Gorenberg M, Ben-Haim S, Jerusalmi J, Liberson A. Lower extremity scintigraphy: the foot and ankle. Semin Nucl Med. 1998;27:62-77. 